\title{
SANS study of mixed cholesteric cellulose nanocrystal - gold nanorod suspensions
}

Received 14th July 2020

Accepted 17th September 2020

$10.1039 / \mathrm{d} 0 \mathrm{cc} 04845 \mathrm{c}$
Jonas Van Rie ${ }^{a}$, Guillermo González-Rubio ${ }^{b}$, Sugam Kumar# , Christina Schütza , Joachim Kohlbrecherd, Michèle Vanroelen ${ }^{\mathrm{e}}$, Tom Van Gerven ${ }^{\mathrm{e}}$, Olivier Deschaumef, Carmen Bartic ${ }^{f}$, Luis M. Liz-Marzán ${ }^{\mathrm{b}, \mathrm{g}}$, German Salazar-Alvarez ${ }^{\mathrm{c}}$ and Wim Thielemans ${ }^{\mathrm{a},{ }^{*}}$
Self-assembly of cellulose nanocrystals (CNCs) doped with anisotropic gold nanorods (AuNRs) was studied by small-angle neutron scattering. Correlation distances and structured domains were analysed to determine the influence of CNC and AuNR concentration on structuring. The transfer of the nematic structure of CNCS to AuNRs was explained in terms of an entropy-driven evolution from an isotropic to a cholesteric phase, with small nematic domains already present in the "isotropic" phase in eqiulibrium with the chiral nematic phase.

Gold nanoparticles (AuNPs) are often selected as suitable partners for co-assembly with CNCs, due to their tuneable morphology and high chemical stability. AuNPs additionally feature the ability to sustain localised surface plasmon resonances, which depend on their morphology, size, and aggregation state, as well as on the presence of molecules in their neighbourhood. ${ }^{1,2}$ This property of AuNPs can explain their allure and wide usage in an array of applications, including in (bio)sensing. ${ }^{3-6}$ The combination of templates and self-assembly techniques has been used to achieve plasmonic nanomaterials with optical activity, ${ }^{7}$ among which cholesterically ordered CNCs offer an attractive route towards unique optical behaviour. ${ }^{8}$ In this scenario, the assembly of AuNPs together with CNCs can further expand the range of applications towards photonic materials, optical devices, and chirally specific catalysts. However, while a large amount of AuNP and CNC synthesis and modification pathways are available, the combination of AuNPs

\footnotetext{
a. Sustainable Materials Lab, Department of Chemical Engineering, KU Leuven, campus Kulak Kortrijk, Etienne Sabbelaan 53, 8500 Kortrijk, Belgium.

b. CICbiomaGUNE, Basque Research and Technology Alliance (BRTA), 20014

Donostia-San Sebastián, Spain

Department of Materials Science and Engineering, Ångström Laboratory, Uppsala University, Box 534, 75121 Uppsala, Sweden

d. Laboratory for Neutron Scattering and Imaging, Paul Scherrer Institute, CH-5232 Villigen, Switzerland

e. Department of Chemical Engineering, KU Leuven, Celestijnenlaan 200F, 3001 Leuven, Belgium

f. Soft Matter and Biophysics Unit, Department of Physics and Astronomy, KU Leuven, Celestijnenlaan 200 D, 3001 Leuven, Belgium

g. Ikerbasque, Basque Foundation for Science

\# Current address: Solid State Physics Division, Bhabha Atomic research Centre, Mumbai, 400 085, India

Electronic Supplementary Information (ESI) available: [details of any supplementary information available should be included here]. See DOI: $10.1039 / \mathrm{d} 0 \mathrm{cc} 04845 \mathrm{c}$
}

and CNCs often lacks optimization of the interactions between components or analysis of a wide range of assembly parameters. Furthermore, a significant amount of the currently available research on AuNP-CNC hybrid materials focuses on dry films and their applications, ${ }^{9-16}$ without a detailed analysis of the pre-drying assembly process. Therefore, a more fundamental knowledge of the interactions between assembling particles and their assembly behaviour in the liquid state are required to improve the quality and reproducibility of the resulting materials.

We present here a detailed study on the influence of the liquid crystalline assembly of CNCs on the organization of dispersed gold nanorods (AuNRs). We used small-angle neutron scattering (SANS), a technique that is particularly powerful to investigate colloidal assemblies comprising distinct materials. Due to the neutron scattering length variation between atomic isotopes, it is possible to selectively observe one specific component within the assembled nanomaterial through appropriate solvent selection (i.e., the arrangement of CNCs and AuNPs can be visualised by matching the solvent scattering length density to either the CNCs or the AuNRs). An elegant example of an arrangement process investigated by contrast matching in SANS can be found in the assembly of AuNPs and amyloid fibrils into ultra-low density aerogels, recently reported by Nyström and co-workers. ${ }^{17}$ This method also permits an analysis of the evolution of the AuNR/CNC system where the CNC concentration is varied. The CNC concentration is known to affect the CNC arrangement in solution, from an isotropic phase (low CNC concentrations) to a cholesteric (higher CNC concentrations) liquid crystalline phase. The SANS experiments conducted and presented in this work used contrast matching to study the structure and spacing of self-assembled AuNRs and CNCs, and allowed a detailed analysis of the structuring evolution of AuNRs as the chiral nematic structure of the CNC matrix was developing. The main goal was to investigate whether and how anisotropic AuNPs position themselves within the cholesteric phase of CNCs. The effect of AuNR inclusions on the CNC structure was also investigated. The CNCs prepared for this study (average length and width: 185 and $6.6 \mathrm{~nm}$ respectively) showed a macroscopic phase separation into an isotropic and a chiral-nematic phase for CNC concentrations above $5 \mathrm{wt} \%$ (ESI, Figures S1 and S2). To study the 
co-assemblies, aqueous CNC suspensions (1.0, 3.0, 5.0, 8.0 and 10.6 wt\%) were mixed with AuNRs (average length $62 \mathrm{~nm}$, width $20.4 \mathrm{~nm}$; ESI, Figures S3.1 and S3.2) and dialysed against mixtures of water and deuterated water (details about the synthesis, dialysis and characterization can be found in ESI). The optimal concentration of AuNRs used throughout the SANS experiments was found to be below 0.03 wt\% (namely, $0.0014,0.007$ and 0.021 wt\%). SANS measurements were performed on the SANS-II instrument at the Swiss spallation neutron source SINQ, Paul Scherrer Institute, Villigen, Switzerland (ESI) ${ }^{18}$ over a q-range of $0.01 \leq \mathrm{q} \leq 1 \mathrm{~nm}^{-1} .19,20$ The isotropic and nematic phases for samples containing $5 \mathrm{wt} \% \mathrm{CNCs}$ and above were measured separately. In the case of anisotropic particles such as CNCs, integration of 2D data maintains structural information, which can be interpreted in terms of liquid crystallinity. ${ }^{21}$ Plots of $\mathrm{I}(\mathrm{q}) \mathrm{q}^{2}$ vs. $\mathrm{q}$, so-called Kratky plots, representing the same data as discussed throughout this paper but with more distinguishable structure peaks can be found in ESI

(Figures S4.3-S4.10). These representations were used to derive the $\mathrm{q}_{\max }$ values through a Lorentz fit, Eq. 1, needed to calculate the correlation distances $(d)$ between nanoparticles (Eq.2):

$$
\begin{gathered}
I(q)=\frac{2 A}{\pi} \frac{w}{4\left(q-q_{\max }\right)^{2}+w^{2}} \\
d=\frac{2 \pi}{q_{\max }}
\end{gathered}
$$

where I(q) is the scattering intensity, $A$ the peak area, $w$ the full width at half-maximum and $\mathrm{q}_{\max }$ the peak position.

We first investigated the role of CNC concentration on the formation of distinct liquid crystal phases. Figure 1a shows SANS spectra for 3, 5 and 8 wt\% CNC suspensions, in the absence of AuNRs. At 3 wt\%, phase separation was not macroscopically visible (Figures 1 and ESI S1), and no clear structure peaks are visible in the SANS spectra. In contrast, the SANS curves for 5 and 8 wt\% CNC display distinct maxima that can be associated with the arrangement of CNCs into a cholesteric liquid crystal phase (lower phase in the solution).
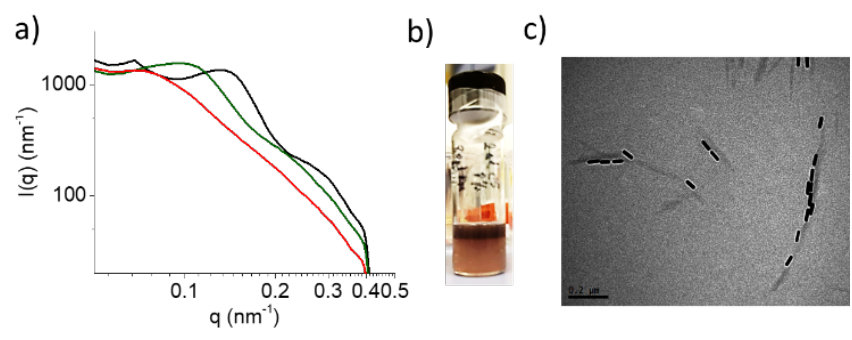

Figure 1. (a) SANS curves of 3 (red), 5 (green) and 8 (black) wt\% CNC suspensions in the absence of AuNRs. The 5 and $8 \mathrm{wt} \%$ curves represent the lower cholesteric phase. The positions of the structure peaks evolve as the CNC concentration changes. (b) An assembly of 0.007 wt\% AuNRs and 8 wt\% CNCs, visually phase separated. (c) Representative TEM micrograph of the AuNR-CNC assembly.

The position of the structure peaks shifts to higher q-values as the concentration increases and the CNC correlation distance decreases, in accordance with recent studies regarding the concentration effect of CNCs on the formation of the liquid crystalline phase. ${ }^{19,20,22}$ The calculated correlation distances were found to decrease from 53 to $43 \mathrm{~nm}$ when the CNC concentration increased from 5 to $8 \mathrm{wt} \%$ (values used as reference values throughout the manuscript).

We then investigated the impact of AuNRs on CNC liquid crystal phase formation. We noticed that the presence of AuNRs did not affect CNC phase separation, as samples containing 5 wt\% CNCs (and higher) still showed macroscopic phase separation (Figure 1b; after mixing the AuNRs and CNCs through vigorous shaking, phase separation reappeared within a couple of minutes). Characterization of AuNR/CNC assemblies via transmission electron microscopy (TEM) showed that AuNRs aligned with the long axis of CNCs (Figure 1c). Most AuNRs were found to interact with CNCs, indicating the existence of attractive interactions. Majoinen et al. observed similar behaviour for cationic spherical AuNPs and CNCs in circular dichroism studies. ${ }^{23}$ Such a phenomenon can be explained by the fact that AuNRs are positively charged while CNCs present negative charges at their surface. AuNRs are functionalised with cetyltrimethylammonium bromide (CTAB), a cationic surfactant that assists their synthesis and maintains colloidal stability, ${ }^{24}$ while CNCs bear sulfate and hydroxyl moieties on their surface (ESI, Experimental Section).

SANS spectra of CNCs in the presence of AuNRs (0.0014 wt\%; AuNR solvent-matched samples) are shown in Figures $\mathbf{2 a}, \mathbf{2 b}$ and ESI Figure S4.1. At 1 and 3 wt\%, the CNCs are still (mainly) randomly oriented, as indicated by featureless SANS curves. In general, the development of structured domains starts at concentrations that are highly dependent on the CNC characteristics and the ionic strength of the solution.22,25,26 Here, for CNC concentrations of $5 \mathrm{wt} \%$ and above, distinct SANS peaks were observed for both the chiral phase (Figure 2a) and the isotropic phase (ESI Figure S4.1). These results are in line with our earlier work which showed that structuring occurs in the isotropic phase before macroscopic phase separation commences. ${ }^{22}$ They also agree with earlier observations in the literature, showing that the development of the cholesteric liquid crystalline phase of CNCs covers a wide concentration range, involving the initial formation of small anisotropic domains within the isotropic phase which slowly evolve towards a macroscopic liquid crystal as the CNC concentration increases. ${ }^{20,22}$ The "isotropic" phase thus exists as a kinetic equilibrium between a large isotropic region with local anisotropic domains, the ratio of which depends on CNC concentration (concept illustrated in Figure 2c). 20, 22, 20,26

The position of the structure peaks shifted when compared to the position of the CNC reference (in the absence of AuNRs, black curve in Figure 2a, and ESI Figure S4.1), indicating that AuNRs interact with CNCs, thereby influencing CNC structuring. The positioning of AuNRs in the CNC matrix pushes the CNCs farther apart. When the CNC concentration was increased from 5 to $8 \mathrm{wt} \%$, the SANS peak shifted towards higher $q$ values, giving rise to a decrease in the correlation distances from 61 to $53 \mathrm{~nm}$ in the chiral nematic phase, and from 59 to $53 \mathrm{~nm}$ in the isotropic phase. Overall, these results indicate that, in the presence of AuNRs, CNCs still form liquid crystal phases but with larger interparticle distances. These 

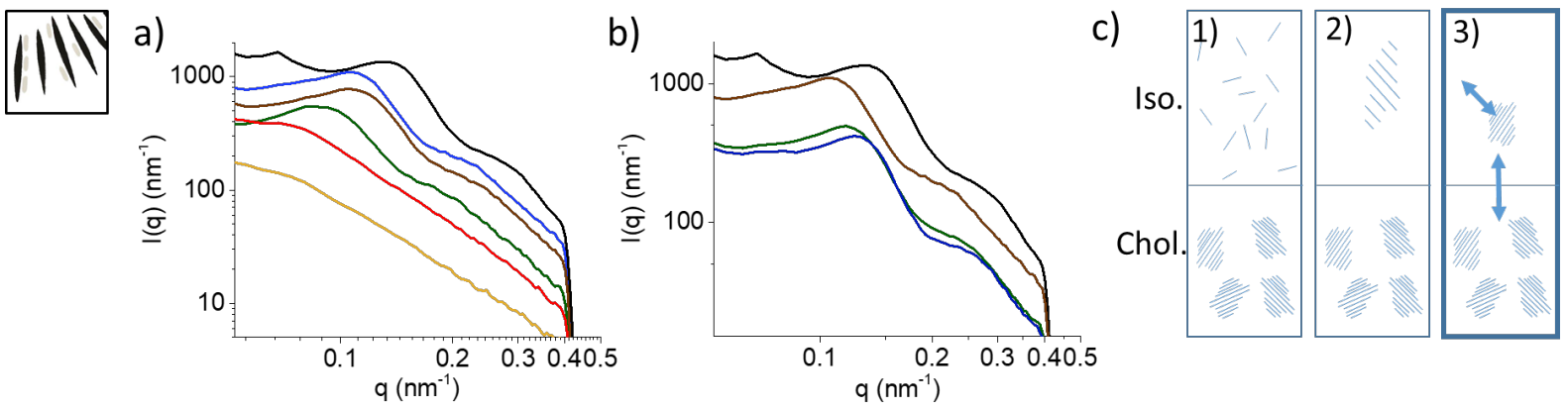

Figure 2. (a) and (b) SANS curves showing the evolution of CNC scattering data for AuNR-CNC assemblies in the nematic (lower) phase, including the 8 (black) wt\% CNC reference SANS curve. (a) Fixed AuNR concentration (0.0014 wt\%) containing 1 (orange), 3 (red), 5 (green), 8 (blue) and 10.6 (brown) wt\% CNCs. SANS curves of the corresponding isotropic (upper) phase can be found in Figure ESI 4.1. (b) Fixed CNC concentration ( 8 wt\%) containing 0.0014 (brown), 0.007 (green) and 0.021 (blue) wt\% AuNRs. (c) Cartoon showing CNCs in the isotropic and cholesteric phase of phase separated suspensions with CNC concentrations above $5 \mathrm{wt} \%$. SANS experiments gave evidence for the existence of small structured domains in the isotropic phase, in equilibrium with the cholesteric phase.

distances decreased again with increasing CNC concentration, in line with pure CNC suspensions. ${ }^{19,20,22}$ A further increase of the CNC concentration, up to $10.6 \mathrm{wt} \%$, only gave a minor $(1 \mathrm{~nm})$ reduction of the correlation distance to $52 \mathrm{~nm}$ in both phases (ESI, Table S1.1).

Literature examples show that plots of correlation distances for CNCs as a function of concentration typically level-out to a minimum value as the $\mathrm{CNC}$ concentration increases, which is also observed here in the presence of AuNRs. ${ }^{19,20,22}$ To further understand the behaviour of the CNC dispersions in the presence of AuNRs, the effect of different AuNR (av. width $20 \mathrm{~nm}$ ) concentrations was investigated by SANS ( 8 wt\% of CNC, Figure $\mathbf{2 b}$ ). In this case, the CNC structure peak shifted to higher $q$-values with increasing AuNR concentration (green and blue curves). For comparison, the SANS curve for 8 wt\% CNCs is also shown (black curve in Figure $2 \mathbf{b}$ ). Interestingly, the position of the structure peak recorded for the $0.021 \mathrm{wt} \%$ AuNR suspension is very close to the position of the pure CNC system ( 0.136 vs. $0.146 \mathrm{~nm}^{-1}$, blue and black curve respectively), with correlation distances of 46 and $43 \mathrm{~nm}$ respectively (Fig. $\mathbf{2 b}$ and ESI Table 1.2). This suggests that an optimal amount of AuNRs exists, where the correlation distance between the CNCs with and without AuNRs can be the same. Indeed, at low AuNR concentration (0.0014 wt\%), the separation distance between CNCs is $53 \mathrm{~nm}$, decreasing to $46 \mathrm{~nm}$ with 0.021 wt\% AuNRs present (ESI Table S1.2).

Until now, we have focused our efforts on investigating the ordering of CNCs in solution and the effect of the presence of AuNRs. Shifting our attention to the structuring of AuNRs in the mixture using CNC solvent-matched experiments (Figure 3), no distinct structure peaks were observed for AuNRs located in the isotropic phase (see ESI, Figure $\mathbf{5} 5$ for a comparison of the SANS plots of the AuNRs in the isotropic and chiral nematic phases). This result may indicate that AuNRs are not, or less, present in the locally aligned domains within the isotropic phase. As alignment is an entropy driven process, one could expect the higher aspect ratio CNCs to be preferentially present in these aligned domains, as this would give the highest entropy gain. Thus, the large CNC translational entropy gain coupled with a larger increase in rotational entropy for the particles remaining in the isotropic region could promote the exclusion of the AuNRs from these local aligned domains. The SANS curves for AuNRs in the CNC matrix displayed structure peaks at higher CNC concentrations (Figure 3a, indicating that AuNRs followed the aligned structuring of the CNCs (co-alignment of AuNRs along the long CNC axis is also illustrated in Figure 1c). As could be expected, the structure peaks of AuNRs are shifted and weaker, when compared to the reference CNC suspensions (Figure 3a). The calculated correlation distances for AuNRs in the CNC suspension are significantly different for 8 and 10.6 wt\% CNCs suspensions; i.e. 56 and $45 \mathrm{~nm}$ respectively (ESI Table 1.3). This is in contrast to what was found earlier for the CNC structure peaks at these concentrations, where the CNC correlation distance seemed to plateau (53 and 52 $\mathrm{nm}$, see also ESI Figure S6a and ESI Table 1.1). At 5 wt\% CNCs, a structure peak for AuNRs was not prominent, which suggests that no significant fraction of AuNRs were incorporated in the chiral nematic phase of the CNCs. This would indicate that AuNRs preferentially locate within the "isotropic" phase (there is a clear colour difference between both phases, with the upper phase more intensely coloured, Fig. 1b) and is in line with the absence of structured AuNRs within aligned domains in this phase. AuNRs may also disrupt structuring of CNCs at concentrations where such local domains are just starting to form and evolve. Finally, the influence of AuNR concentration in $8 \mathrm{wt} \% \mathrm{CNC}$ assemblies on the structuring of AuNRs is shown in Figure $\mathbf{3 b}$. Here, the shift of the AuNR structure peak follows a comparable trend as that found for the CNCs in the same assembly (ESI Figure S6b). At higher AuNR concentrations, the structure peak shifted to higher q-values, approaching the position of 8 wt\% CNC suspensions in the absence of gold. Overall, the characterization of AuNR/CNC systems through SANS experiments and the use of solvent index-matching revealed the interplay between both colloidal nanomaterials. We demonstrated that CNC liquid crystal phase formation was barely affected by the presence of low AuNR concentrations. However, SANS revealed an increase of the correlation distances compared to pure CNC systems, pointing to a closer packing of the CNCs in the liquid crystal phase, most probably resulting from the adsorption of AuNRs on their surface (through Coulombic interactions). In general, increasing $\mathrm{CNC}$ and AuNR concentrations were found to reduce the distances between nanoparticles in the chiral nematic phase (Figure 3c). A preference of lower aspect ratio AuNRs for the isotropic regions was observed, with AuNRs following CNC alignment in the 

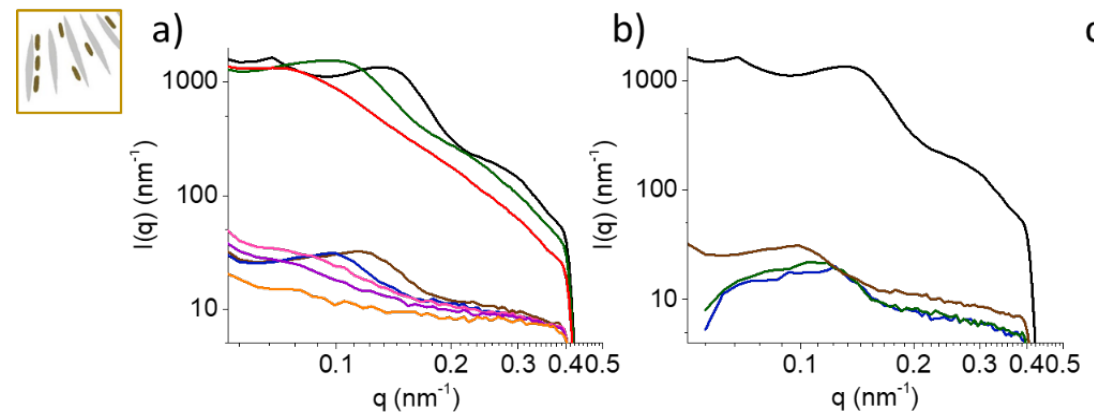

c)

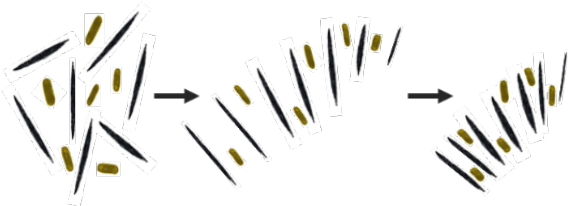

Figure 3. (a) and (b) SANS curves showing the evolution of AuNR scattering data for AuNR-CNC assemblies: Fixed AuNR concentration (0.0014 wt\%) containing 1 (orange), 3 (purple), 5 (pink), 8 (blue), and 10.6 (brown) wt\% CNCs, and 0.0014 wt\% AuNRs. 3 (red), 5 (green), and 8 (black) wt\% CNC reference SANS curves are also shown. (b) Fixed CNC concentration containing 0.0014 (brown), 0.007 (green) and 0.021 (blue) wt\% AuNRs. (c) Cartoon representation of the evolution of the correlation distances and alignment of AuNRs and CNCs at increased concentrations. In general, the correlation distances decrease for AuNRs and CNCs with increasing NP concentration (left to right).

nematic domains. The work presented here reveals that the nematic structure of CNCs can be transferred to AuNRs in solution, and that the lower aspect ratio AuNRs preferentially sequester into the isotropic phase. These results further shed light on the interaction of colloidal AuNPs with CNCs suspensions, thereby providing useful insight toward understanding the formation of hybrid nanomaterials.

\section{Acknowledgements}

Financial support is greatly acknowledged from KU Leuven (WT and CB: grant C14/18/061, WT: grant OT/14/072, OD: grant C14/16/063), Research Foundation Flanders (CB: G0947.17N, WT: G.0C60.13N, JVR: K213816N), and the Swedish Research Council (SK and GSA: 2016-06959). This work uses SANS results obtained at the SANS-II instrument at the Swiss spallation neutron source SINQ, Paul Scherrer Institute, Villigen, Switzerland (proposal 20180361). LML-M acknowledges the Maria de Maeztu Units of Excellence Program from the Spanish State Research Agency (MDM-2017-0720).

\section{Conflicts of interest}

There are no conflicts to declare.

\section{Notes and references}

1 Y.-C. Yeh, B. Creran and V. M. Rotello, Nanoscale, 2012, 4, 18711880.

2 S. Eustis and M. A. El-Sayed, Chem. Soc. Rev., 2006, 35, 209-217.

3 J. M. Romo-Herrera, R. A. Alvarez-Puebla and L. M. Liz-Marzán, Nanoscale, 2011, 3, 1304-1315.

4 L. M. Liz-Marzán, Langmuir, 2006, 22, 32-41.

5 C. Hamon, S. Novikov, L. Scarabelli, L. Basabe-desmonts and L. M. Liz-marzán, ACS Nano, 2014, 8, 10694-10703.

6 H. Okamoto and K. Imura, Prog. Surf. Sci., 2009, 84, 199-229.

7 J. Van Rie and W. Thielemans, Nanoscale, 2017, 9, 8525-8554.

8 A. Guerrero-Martínez, J. L. Alonso-Gómez, B. Auguié, M. M. Cid and L. M. Liz-Marzán, Nano Today, 2011, 6, 381-400.

9 M. Schlesinger, W. Y. Hamad and M. J. MacLachlan, Soft Matter, 2015, 11, 4686-4694.
10 A. Lukach, H. Thérien-Aubin, A. Querejeta-Fernández, N. Pitch, G. Chauve, M. Méthot, J. Bouchard and E. Kumacheva, Langmuir, 2015, 31, 5033-5041.

11 G. Chu, X. Wang, T. Chen, W. Xu, Y. Wang, H. Song and Y. Xu, J. Mater. Chem. C, 2015, 3, 3384-3390.

12 D. Qu, J. Zhang, G. Chu, H. Jiang, C. Wu and Y. Xu, J. Mater. Chem. C, 2016, 4, 1764-1768.

13 Y. Wang, J. He, C. Liu, W. H. Chong and H. Chen, Angew. Chemie Int. Ed., 2015, 54, 2022-2051.

14 Z. Cheng, Y. Ma, L. Yang, F. Cheng, Z. Huang, A. Natan, H. Li, Y. Chen, D. Cao, Z. Huang, Y. Wang, Y. Liu, R. Yang and H. Zhu, Adv. Opt. Mater., 2019, 0, 1801816.

15 Z. Hu, Q. Meng, R. Liu, S. Fu and L. A. Lucia, ACS Sustain. Chem. Eng., 2017, 5, 1601-1609.

16 C. R. Cabreira and F. F. Camilo, Cellulose, 2020, 27, 3919-3929.

17 G. Nyström, M. P. Fernández-Ronco, S. Bolisetty, M. Mazzotti and R. Mezzenga, Adv. Mater., 2016, 28, 472-478.

18 P. Strunz, K. Mortensen and S. Janssen, Phys. B Condens. Matter, 2004, 350, E783-E786.

19 M. Uhlig, A. Fall, S. Wellert, M. Lehmann, S. Prévost, L. Wågberg, R. von Klitzing and G. Nyström, Langmuir, 2016, 32, 442-450.

20 C. Schütz, M. Agthe, A. B. Fall, K. Gordeyeva, V. Guccini, M. Salajková, T. S. Plivelic, J. P. F. Lagerwall, G. Salazar-Alvarez and L. Bergström, Langmuir, 2015, 31, 6507-6513.

$21 \mathrm{Y}$. B. Melnichenko, Small-angle scattering from confined and interfacial fluids: Applications to Energy Storage and Environmental Science, Springer International Publishing, Switzerland, 2016.

22 J. Van Rie, C. Schütz, A. Gençer, S. Lombardo, U. Gasser, S. Kumar, G. Salazar-Alvarez, K. Kang and W. Thielemans, Langmuir, 2019, 35, 2289-2302.

23 J. Majoinen, J. Hassinen, J. S. Haataja, H. T. Rekola, E. Kontturi, M. A. Kostiainen, R. H. A. Ras, P. Törmä and O. Ikkala, $A d v$. Mater., 2016, 28, 5262-5267.

24 S. Gómez-Graña, F. Hubert, F. Testard, A. Guerrero-Martínez, I. Grillo, L. M. Liz-Marzán and O. Spalla, Langmuir, 2012, 28, 14531459.

25 C. Schütz, J. R. Bruckner, C. Honorato-Rios, Z. Tosheva, M. Anyfantakis and J. P. F. Lagerwall, Crystals, 2020, 10, 199.

26 Y. Liu, M. Agthe, M. Salajková, K. Gordeyeva, V. Guccini, A. Fall, G. Salazar-Alvarez, C. Schütz and L. Bergström, Nanoscale, 2018, 10, 18113-18118. 


\title{
Electronic Supporting Information
}

\section{SANS study of mixed cholesteric cellulose nanocrystal - gold nanorod suspensions}

\author{
Jonas Van Rie ${ }^{a}$, Guillermo González-Rubio ${ }^{b}$, Sugam Kumar ${ }^{c \#}$, Christina Schütz ${ }^{a}$, Joachim \\ Kohlbrecher ${ }^{d}$, Michèle Vanroelen ${ }^{e}$, Tom Van Gerven ${ }^{e}$, Olivier Deschaume ${ }^{f}$, Carmen Bartic ${ }^{f}$, \\ Luis M. Liz-Marzán ${ }^{\mathrm{b}, \mathrm{g}}$, German Salazar-Alvarez ${ }^{\mathrm{c}}$ and Wim Thielemans ${ }^{\mathrm{a},{ }^{*}}$
}

\begin{abstract}
a. Sustainable Materials Lab, Department of Chemical Engineering, KU Leuven, campus Kulak Kortrijk, Etienne Sabbelaan 53, 8500 Kortrijk, Belgium.

b. CICbiomaGUNE, Basque Research and Technology Alliance (BRTA), 20014 Donostia-San Sebastián, Spain

c. Department of Materials Science and Engineering, Ångström Laboratory, Uppsala University, Box 534, 75121 Uppsala, Sweden

d. Laboratory for Neutron Scattering and Imaging, Paul Scherrer Institute, $\mathrm{CH}-5232$ Villigen, Switzerland.

e. Department of Chemical Engineering, KU Leuven, Celestijnenlaan 200 F, 3001 Leuven, Belgium

f. Soft Matter and Biophysics Unit, Department of Physics and Astronomy, KU Leuven, Celestijnenlaan 200 D, 3001 Leuven, Belgium

g. Ikerbasque, Basque Foundation for Science

\# Current address: Solid State Physics Division, Bhabha Atomic research Centre, Mumbai, 400 085, India
\end{abstract}




\section{Experimental Section}

CNC synthesis: Standard procedures were followed to obtain a cellulose nanocrystal suspension. ${ }^{1}$ Cotton wool was hydrolysed in sulfuric acid ( $64 \mathrm{wt} \%)$ for 40 minutes at $45^{\circ} \mathrm{C}$. After centrifugation and dialysis, excess $\mathrm{H}^{+}$and $\mathrm{OH}^{-}$ions were removed through mixing with Amberlite $\mathrm{MB}-6113$ ion-exchange resin. A stable CNC suspension was subsequently obtained after removal of the resin by filtration. Excess water was evaporated under ambient conditions to a concentration of $10.6 \mathrm{wt} \%$. This cellulose nanocrystal suspension was subsequently carefully diluted. To illustrate the development of the different phases as a function of cellulose nanocrystal concentration, a picture of $1-10 \mathrm{wt} \% \mathrm{CNC}$ suspensions is shown in Figure SI-1. As can be seen in Figure SI1, macroscopic phase separation into an isotropic and chiral-nematic phase was observed for CNC concentrations of $5 \mathrm{wt} \%$ and higher. A Netzsch F3 Tarsus gravimetry instrument was used to determine the concentrations of CNC suspensions. The procedure involved heating of the samples to $85{ }^{\circ} \mathrm{C}$ at $10{ }^{\circ} \mathrm{C}$ per minute, with a subsequent isothermal period of 30 minutes, ending with another heating period at $10^{\circ} \mathrm{C}$ per minute to a final $150{ }^{\circ} \mathrm{C}$. The $\mathrm{CNCs}$ were furthermore characterized by atomic force microscopy (AFM) to have an average length of $185 \pm 92 \mathrm{~nm}$ and width of $6.6 \pm 2.4 \mathrm{~nm}$.

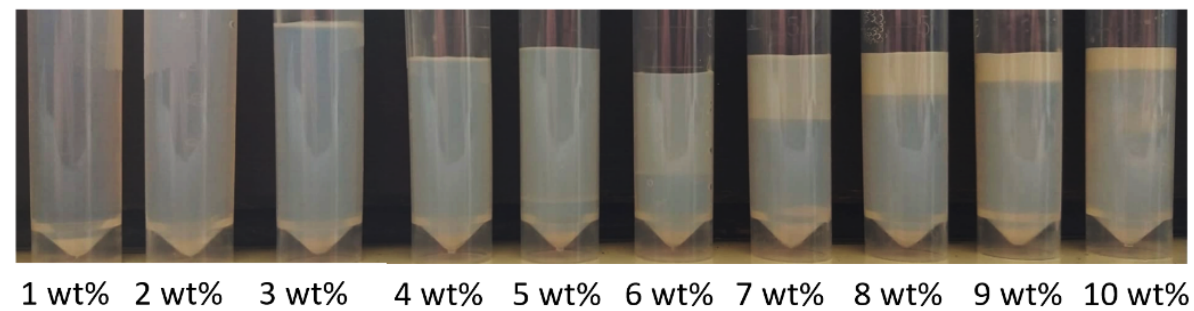

Figure S1. Cellulose nanocrystal suspensions. From 5 wt\%, macroscopic phase separation is visible.

AuNR synthesis: AuNRs were synthesised based on the seed-mediated growth procedure described by Scarabelli et al. ${ }^{2}$ All solutions were prepared with fresh milliQ water $(18.2 \mathrm{M} \Omega \mathrm{cm})$. Seed solution. Twenty five $\mu$ l of a $0.05 \mathrm{M} \mathrm{HAuCl}_{4}$ solution was added to $4.7 \mathrm{ml}$ of a $0.1 \mathrm{M} \mathrm{CTAB}$ solution at $30^{\circ} \mathrm{C}$, turning the solution yellow. This was stirred at $300 \mathrm{rpm}$ for $5 \mathrm{~min}$. Next, a $10 \mathrm{mM} \mathrm{NaBH}_{4}$ solution was freshly prepared and $300 \mu \mathrm{L}$ of this solution was added while stirring at $1200 \mathrm{rpm}$, turning the colour of the seed brown. Stirring was reduced to $400 \mathrm{rpm}$ after 20 seconds, and stopped after a couple of minutes AuNR Growth: $900 \mathrm{mg}$ CTAB was dissolved in $50 \mathrm{ml}$ milliQ water at $30^{\circ} \mathrm{C}$. After the solution turned completely transparent, $50 \mathrm{mg} 5$-bromosalicylic acid was added while stirring slowly. When everything was dissolved, $480 \mu \mathrm{l}$ of a $0.01 \mathrm{M} \mathrm{AgNO}_{3}$ solution was added under stirring at $300 \mathrm{rpm}$. This solution was stirred for another 15 minutes at $300 \mathrm{rpm}$, after which $500 \mu \mathrm{l}$ of a $0.05 \mathrm{M} \mathrm{HAuCl}_{4}$ solution was added. This quickly turned the solution dark orange. The solution was further stirred at $300 \mathrm{rpm}$ during 1 hour and the colour slowly evolved towards light yellow (until the absorption intensity of the peak at $390 \mathrm{~nm}$ decreased to $0.5-0.6$ as measured in a cuvette of optical pathlength of $1 \mathrm{~cm}$ ). Next, $130 \mu \mathrm{l}$ of a $17.4 \mathrm{mg} / \mathrm{mL}$ ascorbic acid solution was added while stirring at $1200 \mathrm{rpm}$. Upon addition, the colour of the solution turned almost immediately transparent. $80 \mu \mathrm{L}$ of a freshly prepared seed solution was quickly added while stirring at $1200 \mathrm{rpm}$. After 30 seconds, stirring was stopped and the solution was left to grow for a couple of hours. Finally, The AuNRs were centrifuged for 30 minutes at $6000 \mathrm{rpm}$. The gold concentration in the aqueous phase was measured by Inductively Coupled Plasma Mass Spectrometry (ICP-MS, Perkin Elmer ELAN DRC-e). Aqueous samples were diluted in $10 \mathrm{~mL} 2 \% \mathrm{HNO}_{3}$ and Lutetium was used as an internal standard. If intermediate dilutions were required, Yttrium was added as a second internal standard to check the consistency of these dilutions. The dimensions of the AuNRs were determined by AFM, which gave an average length of $62 \pm 4.8 \mathrm{~nm}$ and average width of $20.4 \pm 3.3 \mathrm{~nm}$. 
AuNR-CNC system. AuNR solutions of different concentrations (i.e., $0.0014,0.007$ and 0.021 wt\%) were mixed with the aqueous CNC suspensions $(1,3,5,8$ and $10.6 \mathrm{wt} \%)$ and dialysed against mixtures of water and deuterated water. Dialysis tubes were filled with $1 \mathrm{~mL}$ of each sample, and left to dialyse in $10 \mathrm{~mL}$ of a $\mathrm{D}_{2} \mathrm{O}: \mathrm{H}_{2} \mathrm{O}$ mixture. After two days, the mixture of $\mathrm{D}_{2} \mathrm{O}: \mathrm{H}_{2} \mathrm{O}$ mixture was refreshed. A 35:65 $\mathrm{D}_{2} \mathrm{O}: \mathrm{H}_{2} \mathrm{O}$ mixture was used to prepare the suspension with the solvent contrast matched to CNCs in the assembly and for the independent visualisation of the AuNRs. ${ }^{3} \mathrm{~A} 72: 28 \mathrm{D}_{2} \mathrm{O}: \mathrm{H}_{2} \mathrm{O}$ mixture was used to match the solvent to AuNRs and visualize the CNCs in the AuNR-CNC solutions. ${ }^{4}$ Pure CNC suspensions (references) were dialysed against pure $\mathrm{D}_{2} \mathrm{O}$.

\section{Characterization Techniques}

SANS experiments: SANS spectra were recorded on the SANS-II instrument at the Swiss spallation neutron source SINQ, Paul Scherrer Institute, Villigen, Switzerland. ${ }^{5}$ The samples were measured at a $6 \mathrm{~m}$ sample-detector distance, lambda $0.73 \mathrm{~nm}$ and a $3 \mathrm{~m}$ sample-detector distance, lambda 0.55 . The set-up had a ${ }^{3} \mathrm{He}$ detector with $128 \times 128$ pixels. One $\mathrm{mm}$ path length quartz glass capillaries were used for all measurements. The samples were loaded in a sample holder and measured in the same run. Based on standard procedures, all obtained data were calibrated for detector nonlinearity using the incoherent scattering from $1 \mathrm{~mm}$ thick $\mathrm{D}_{2} \mathrm{O}$ samples. The scanned q-range was $0.01 \leq \mathrm{q} \leq 1 \mathrm{~nm}^{-1}$ to study the arrangement of gold nanoparticles in the CNC matrix. ${ }^{6,7}$ BerSANS software was used to subtract dark background due to electronic noise/stray neutrons and the background due to the sample environment to obtain the scattering signal of the sample. BerSANS was furthermore used for initial data reduction and to obtain values for the transmissions of all measured samples. ${ }^{8}$ Further data reduction was done using GRASP software ( $a$ Matlab $^{\mathrm{TM}}$ script developed by Institut Laue-Langevin for analysis and reduction of data produced by SANS experiments) to obtain the final 1D scattering patterns.

\section{$\underline{\text { AFM characterization }}$}

CNCs: On a freshly by scotch-tape cleaved mica surface (NanoAndMore GMBH) $20 \mu \mathrm{L}$ poly-L-lysine solution (Sigma Aldrich, $0.1 \% \mathrm{w} / \mathrm{v}$ in water) was deposited for 3 minutes, subsequently rinsed with deionized water and dried with compressed air. Following, $20 \mu \mathrm{L}$ of a CNC dispersion $(0.001$ wt\%) was placed on the treated surface for 3 minutes, then rinsed with deionized water and dried with compressed air. The sample were left in a vacuum oven at $40^{\circ} \mathrm{C}$ overnight. A Multi-mode V AFM (Digital instruments Nanoscope Veeco) was used in tapping mode and AFM probes from Budget Sensors (Tap300 Al-G, resonance frequency $300 \mathrm{kHz}$ and force constant $40 \mathrm{~N} / \mathrm{M}$ ) were used to image the samples. The length of 300 particles was measured manually using image ${ }^{9}$ and the mean length and standard deviation as well as a log-normal distribution were determined. The width was extracted as the height for at least 450 points on representative particles. 


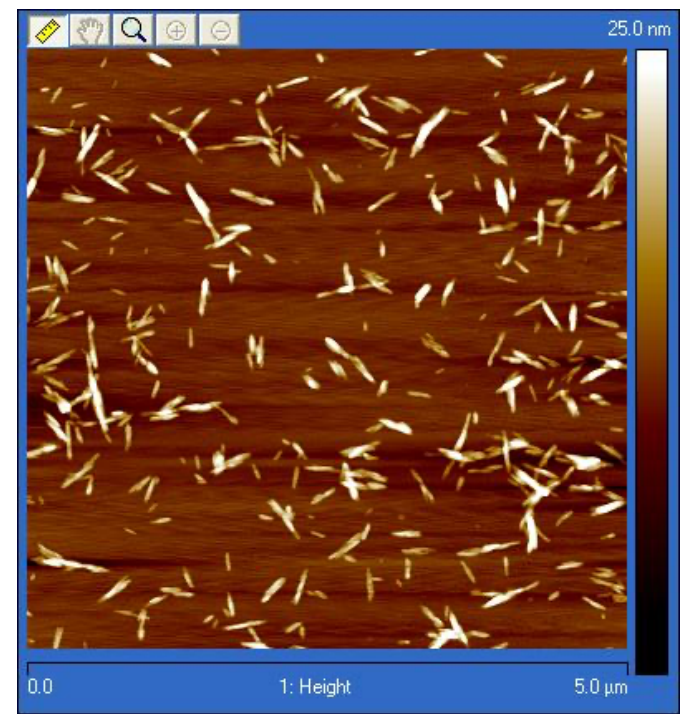

Figure S2.1 AFM images of CNCs.
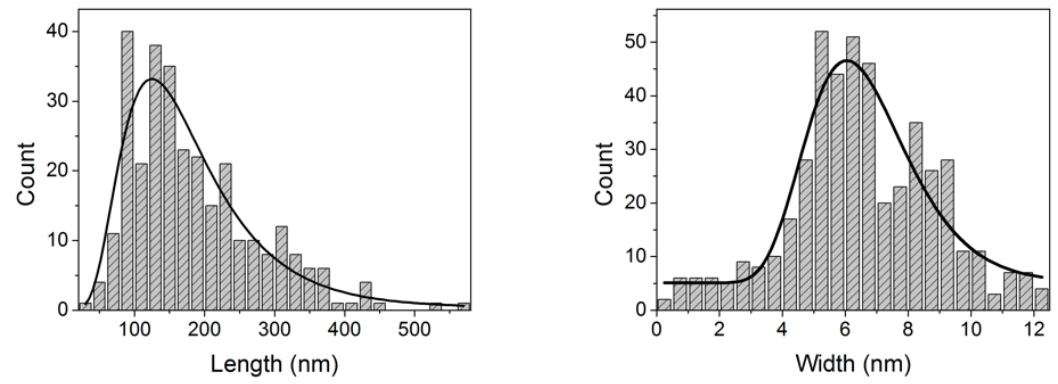

Figure S2.2. Histogram of the length (left) and width (right) distributions of CNCs measured from AFM images.

AuNRs. An Agilent 5500 AFM system with MSNL-F cantilevers ( $f=110=120 \mathrm{kHz}, \mathrm{k}=0.6 \mathrm{~N} / \mathrm{m}$, average tip radius of $2=12 \mathrm{~nm}$ ) was used for topographical imaging of the particles in intermittent contact (AAC) mode. The AFM topography images were leveled, line-corrected and measured (height profiles) using Gwyddion, ${ }^{10}$ a free and open-source SPM (scanning probe microscopy) data visualization and analysis program.
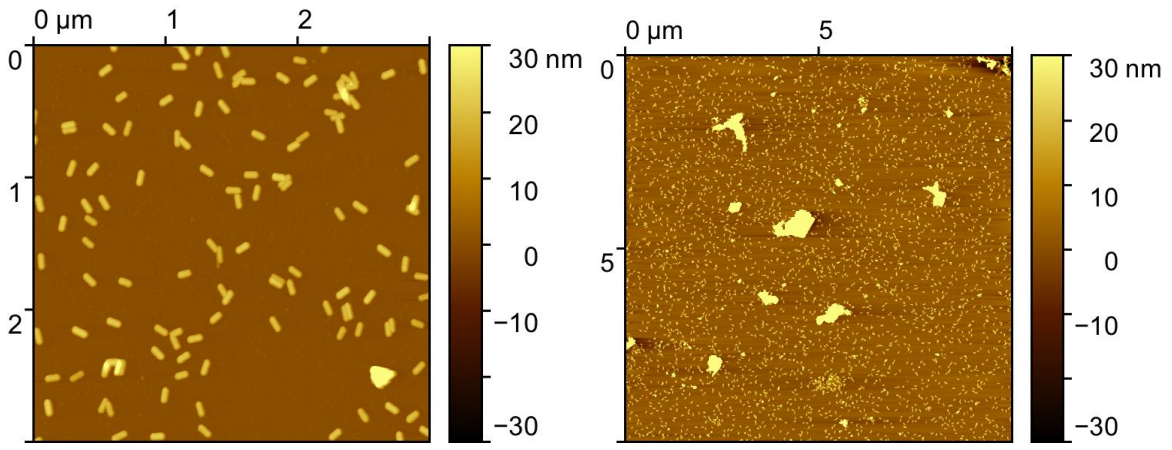

Figure S 3.1. AFM images of AuNRs. 

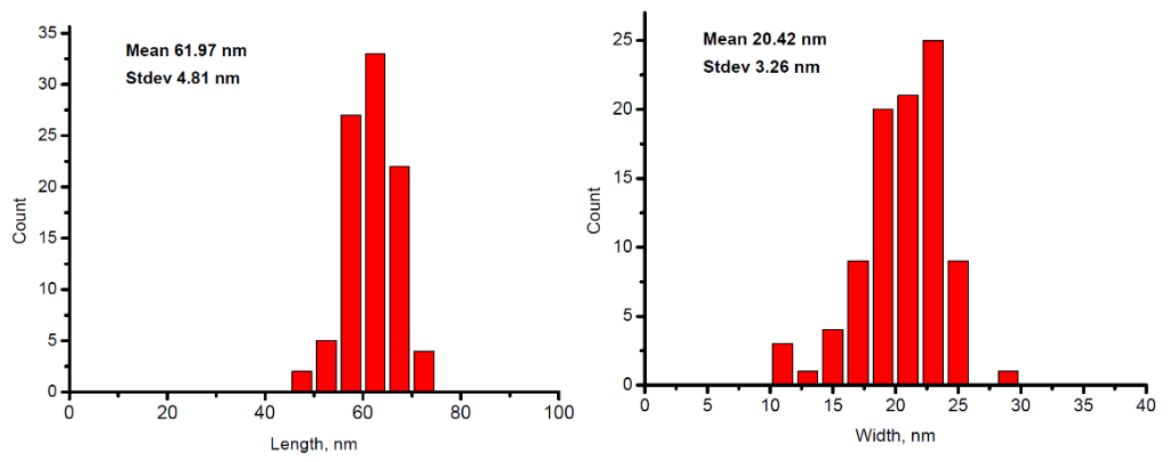

Figure S3.2. Mean length (left) and width (right) histograms of AuNRs.

TEM characterization of AUNR-CNC: Low magnification TEM images were obtained with a JEOL JEM1400 PLUS transmission electron microscope, operating at an acceleration voltage of $120 \mathrm{kV}$. Carboncoated 400 square mesh copper grids were used. 


\section{SANS Data Analysis:}

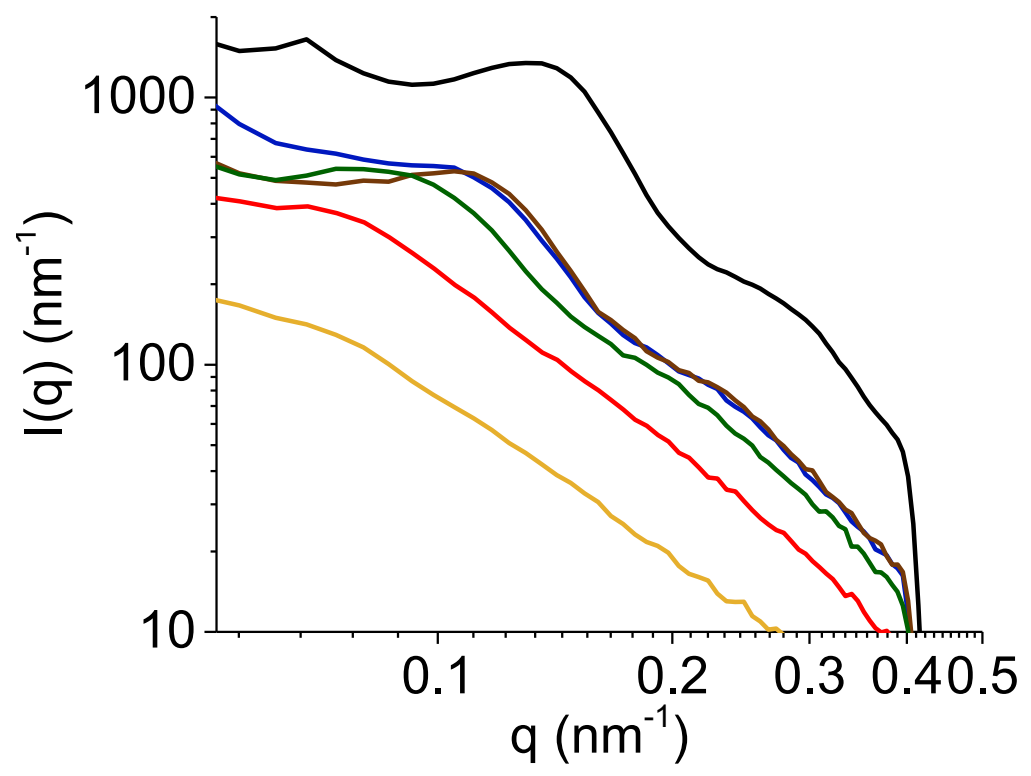

Figure S4.1. SANS curves showing the evolution of CNC scattering data at fixed AuNR concentration (0.0014 wt\%) for AuNRCNC assemblies in the isotropic (upper) phase, containing 1 (orange), 3 (red), 5 (green), 8 (blue) and 10.6 (brown) wt\% CNCs. An 8 (black) wt\% CNC reference SANS curve is also shown.

\section{$\underline{\text { SANS curves with Lorentz fits and Kratky representations }}$}

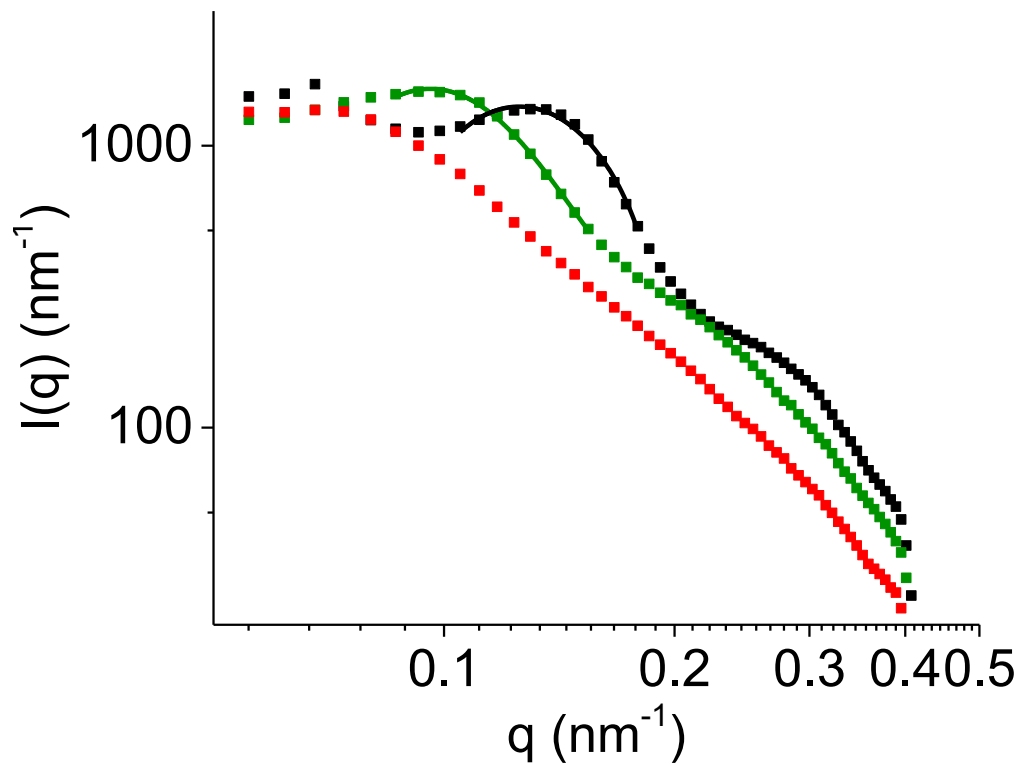

Figure S4.2. SANS curves and Lorentz fits of 3 (red), 5 (green) and 8 (black) wt\% CNC suspensions. 


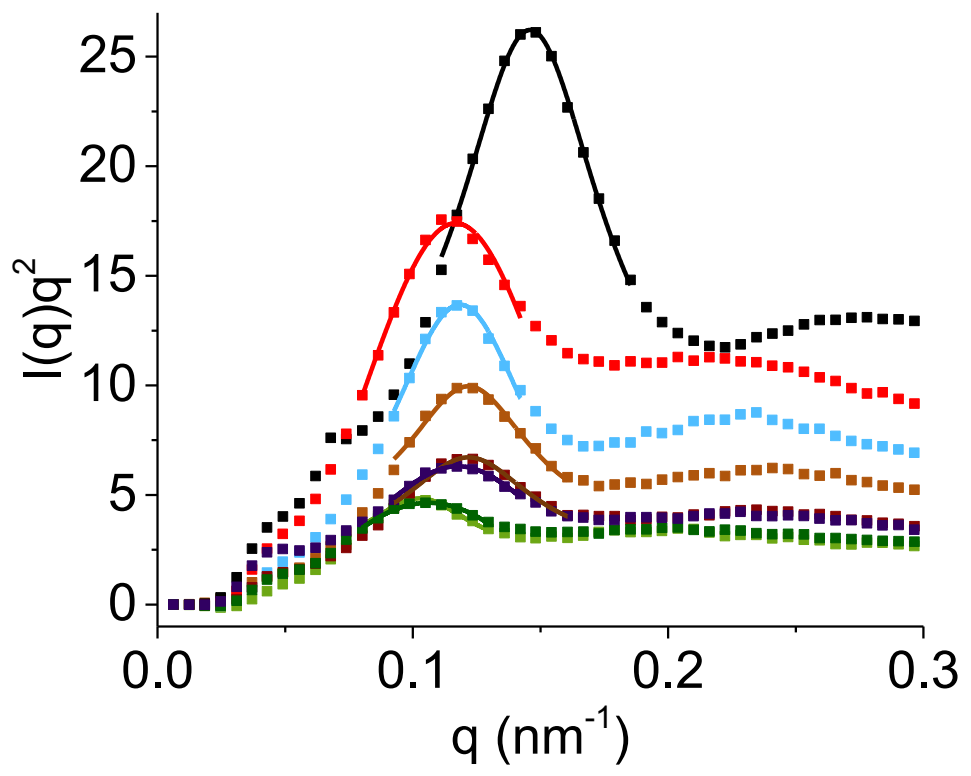

Figure S4.3. Kratky plots and Lorentz fits of the AuNR-CNC systems (matching for gold) containing 5 (green), 8 (blue) and 10.6 (brown) wt\% CNCs and 0.0014 wt\% AuNRs (dark green, blue and brown curves represent the isotropic phase; light green, blue and brown curves the chiral nematic phase). Five (red) and 8 (black) wt\% CNC curves in the absence of AuNRs are shown for comparison.

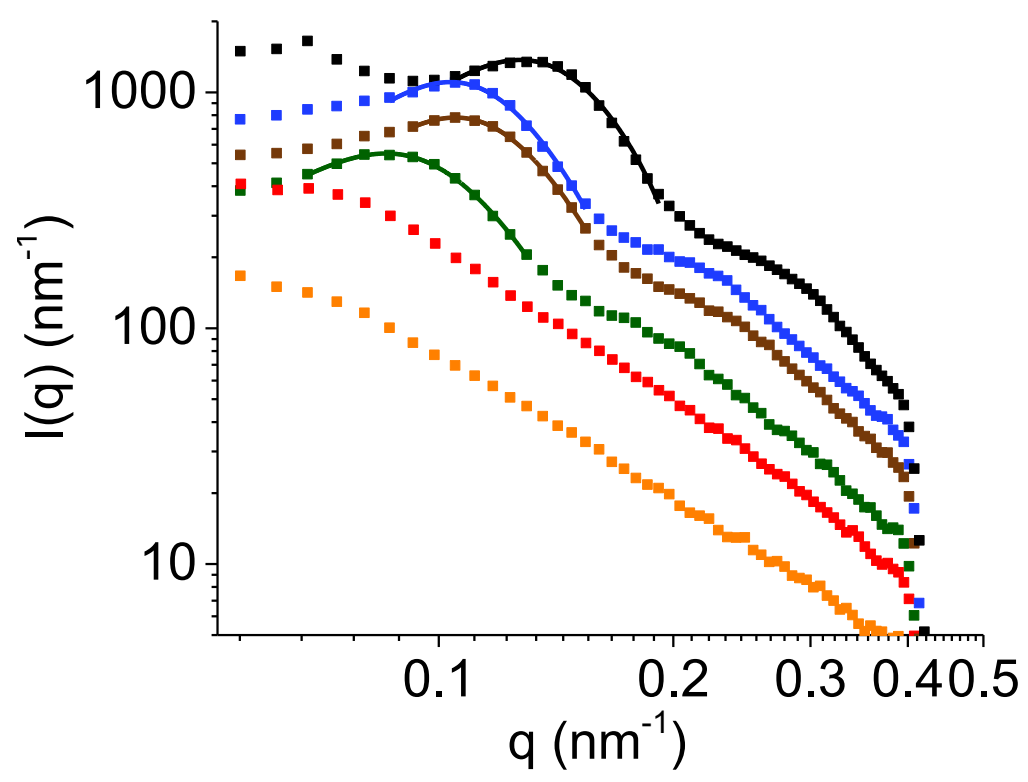

Figure S4.4. SANS plots of the AuNR-CNC systems in the nematic liquid crystalline phase (matching for gold) containing 1 (orange), 3 (red), 5 (green), 8 (blue) and 10.6 (brown) wt\% CNCs and 0.0014 wt\% AuNRs, showing Lorentz fits. An 8 (black) $w t \%$ CNC curve in the absence of AuNRs is shown for comparison. 

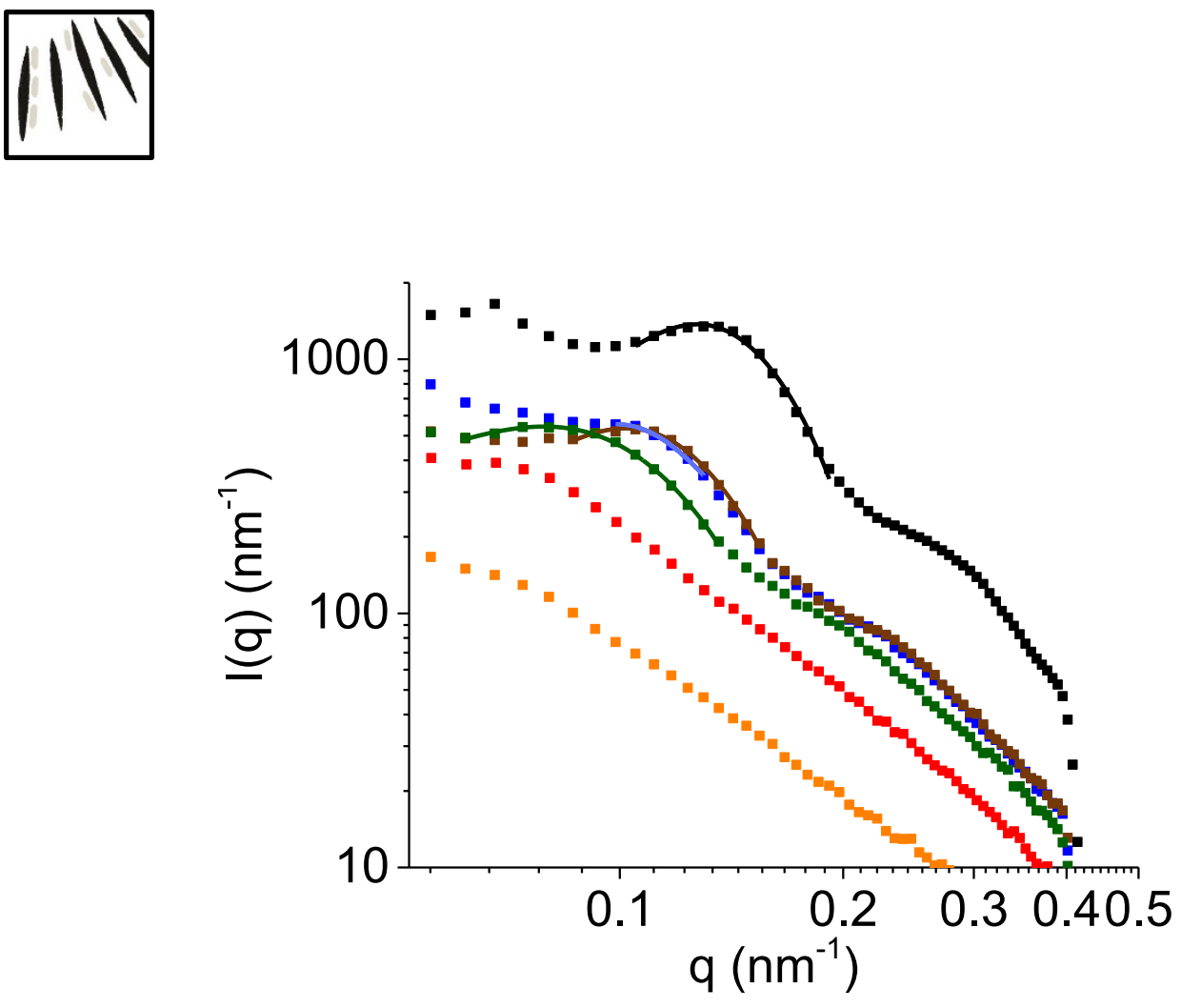

Figure S4.5. SANS plots of the AuNR-CNC systems in the isotropic phase (matching for gold) containing 1 (orange), 3 (red), 5 (green), 8 (blue) and 10.6 (brown) wt\% CNCs and 0.0014 wt\% AuNRs, showing Lorentz fits. An 8 (black) wt\% CNC curve in the absence of AuNRs is shown for comparison.

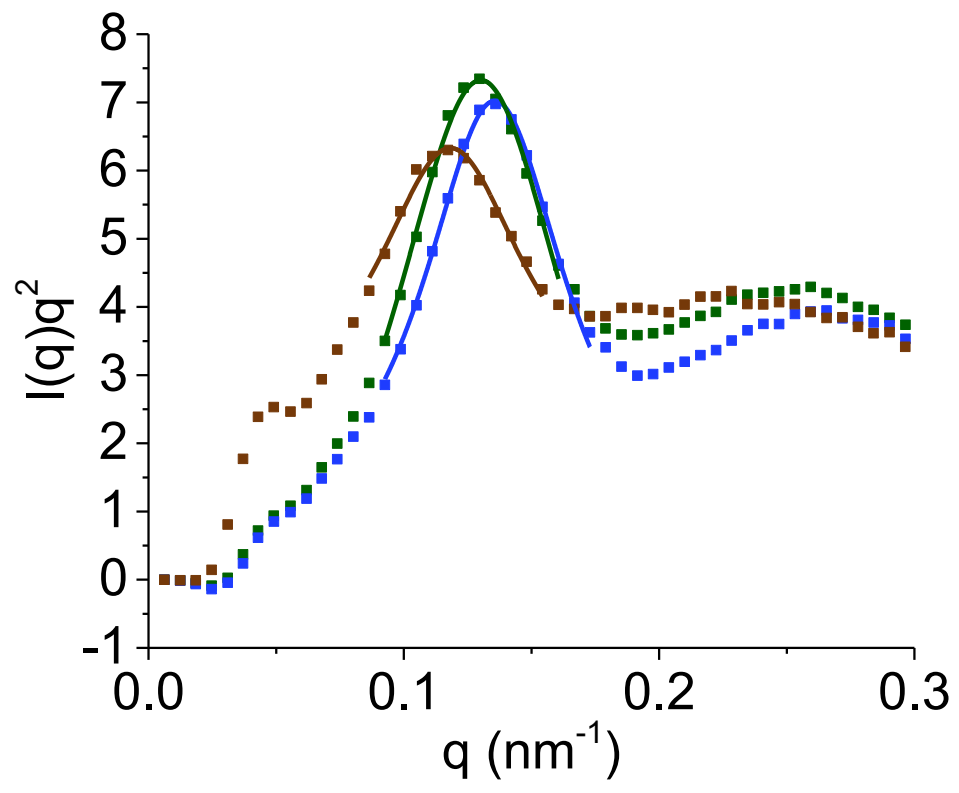

Figure S4.6. Kratky plots of the AuNR-CNC systems (matching for gold) containing 0.0014 (brown), 0.007 (green) and 0.021 (blue) wt\% of AuNRs and 8 wt\% of CNCs, showing Lorentz fits. 

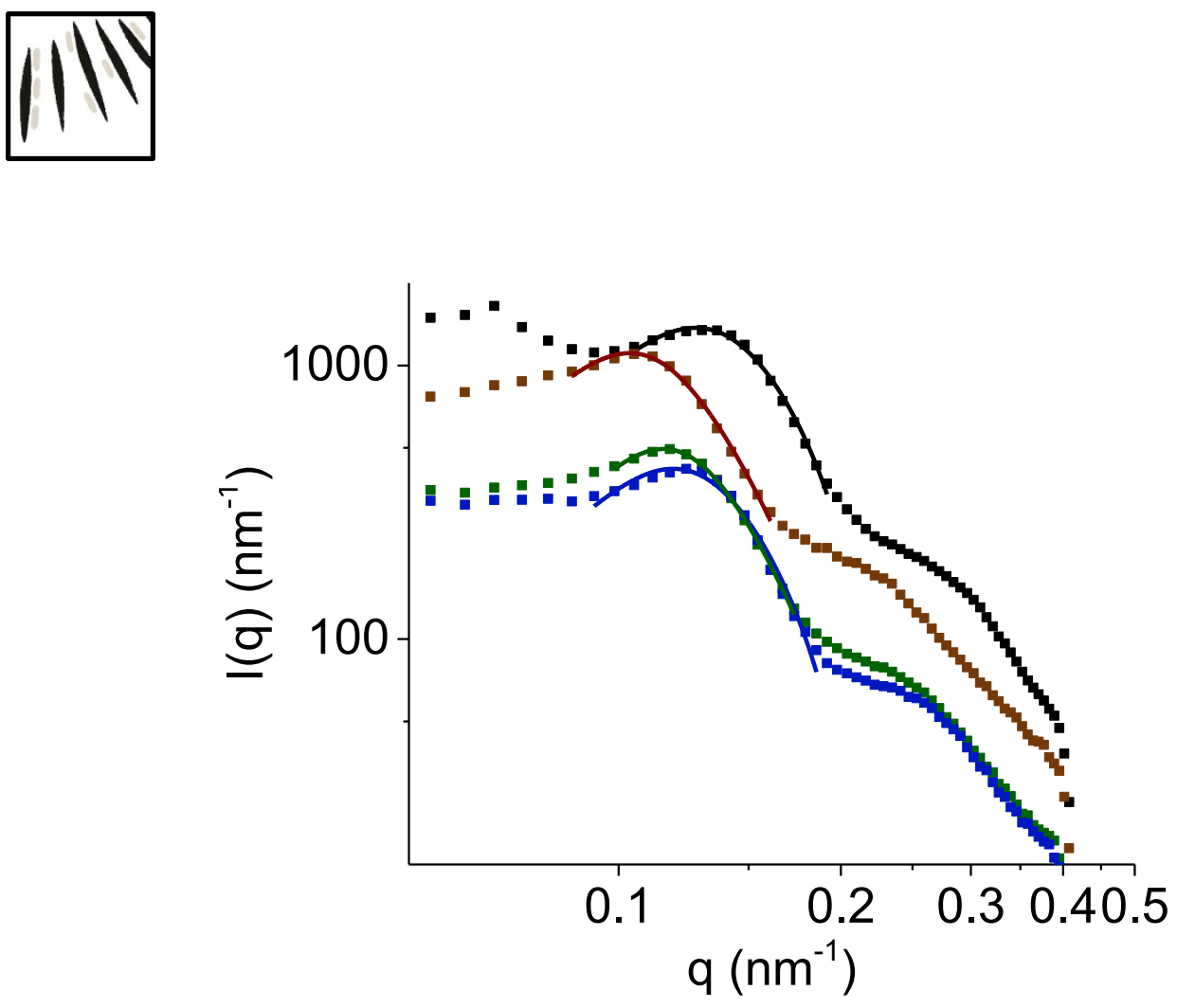

Figure S4.7. SANS plots of the AuNR-CNC systems (matching for gold) containing 0.0014 (brown), 0.007 (green) and 0.021 (blue) wt\% of AuNRs and 8 wt\% of CNCs, showing Lorentz fits. An 8 (black) wt\% CNC curve in the absence of AuNRs is shown for comparison. 

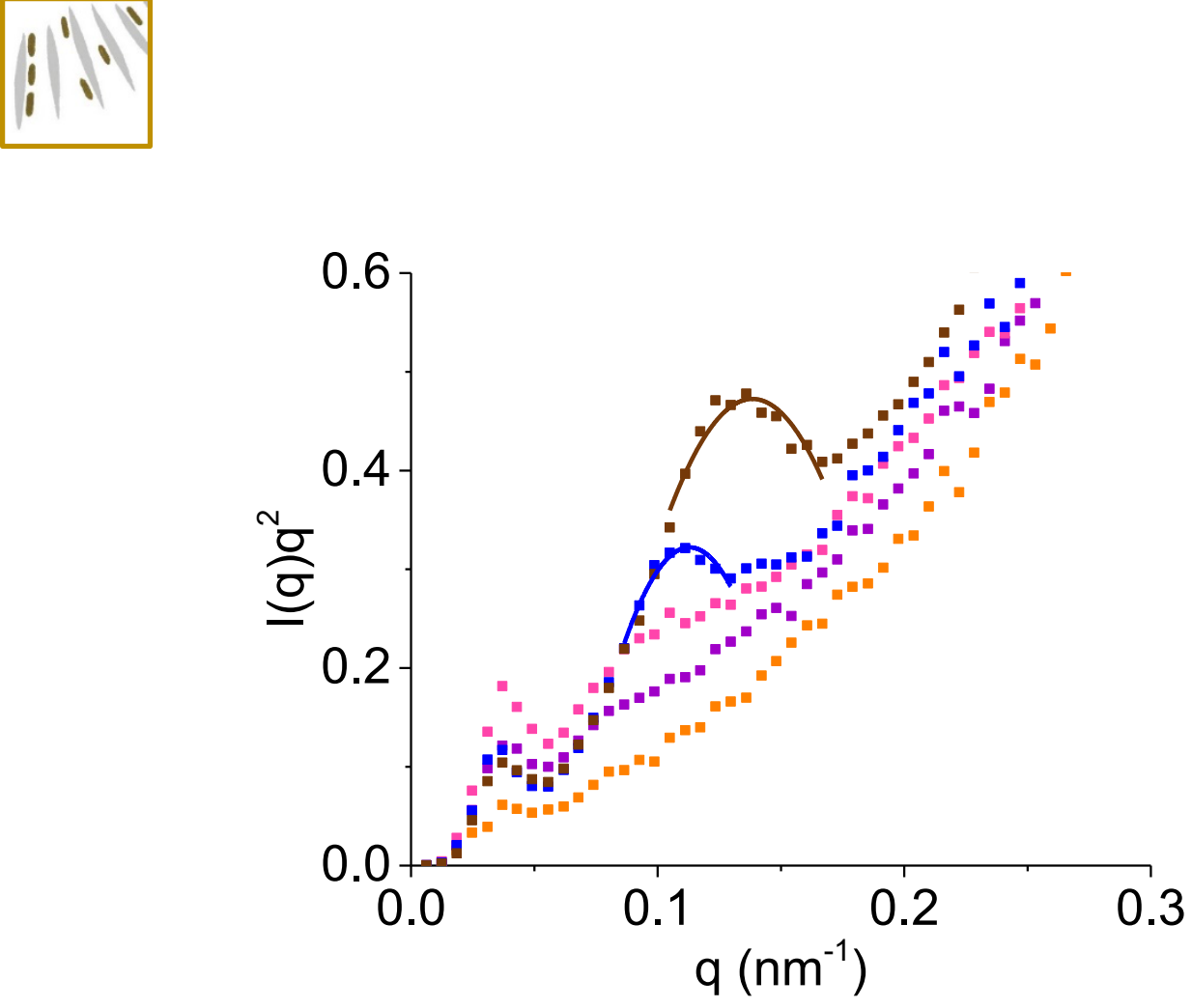

Figure S4.8. Kratky plots of the AuNR-CNC systems (matching for CNCs) containing 1 (orange), 3 (purple), 5 (pink), 8 (blue) and 10.6 (brown) wt\% CNCs and 0.0014 wt\% AuNRs, showing Lorentz fits.

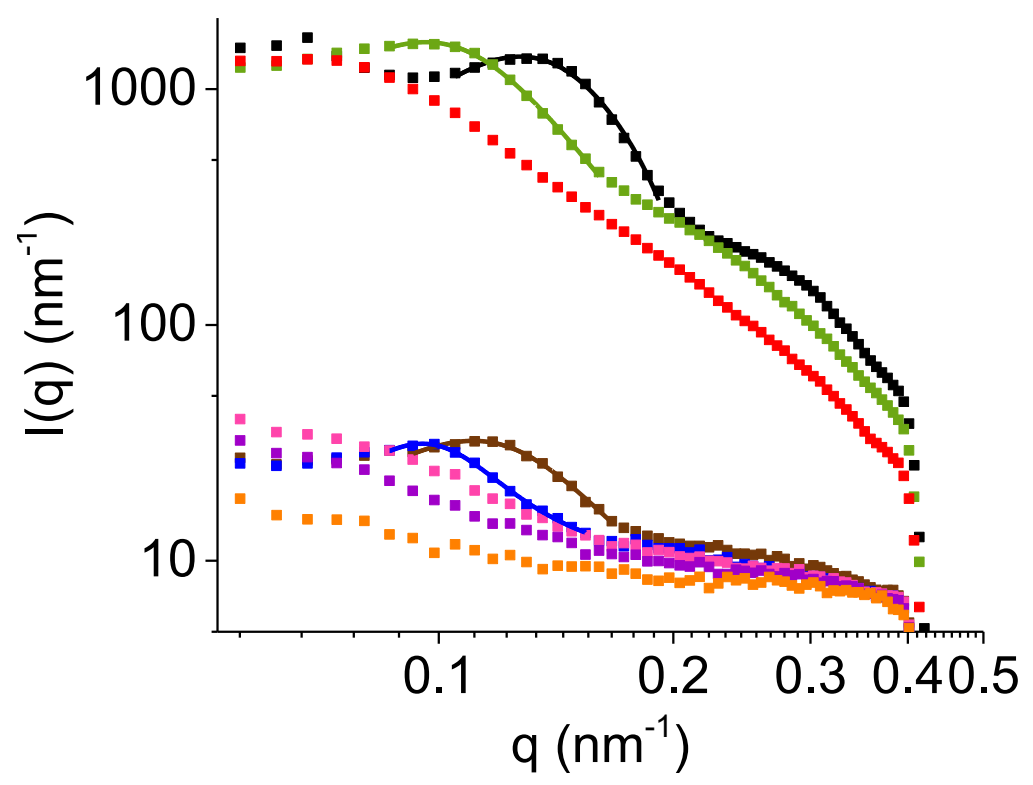

Figure S4.9. Kratky plots of the AuNR-CNC systems (matching for CNCs) containing 1 (orange), 3 (purple), 5 (pink), 8 (blue) and 10.6 (brown) wt\% CNCs and 0.0014 wt\% AuNRs, showing Lorentz fits. Three (red), 5 (green) and 8 (black) wt\% CNC curves in the absence of AuNRs are shown for comparison. 


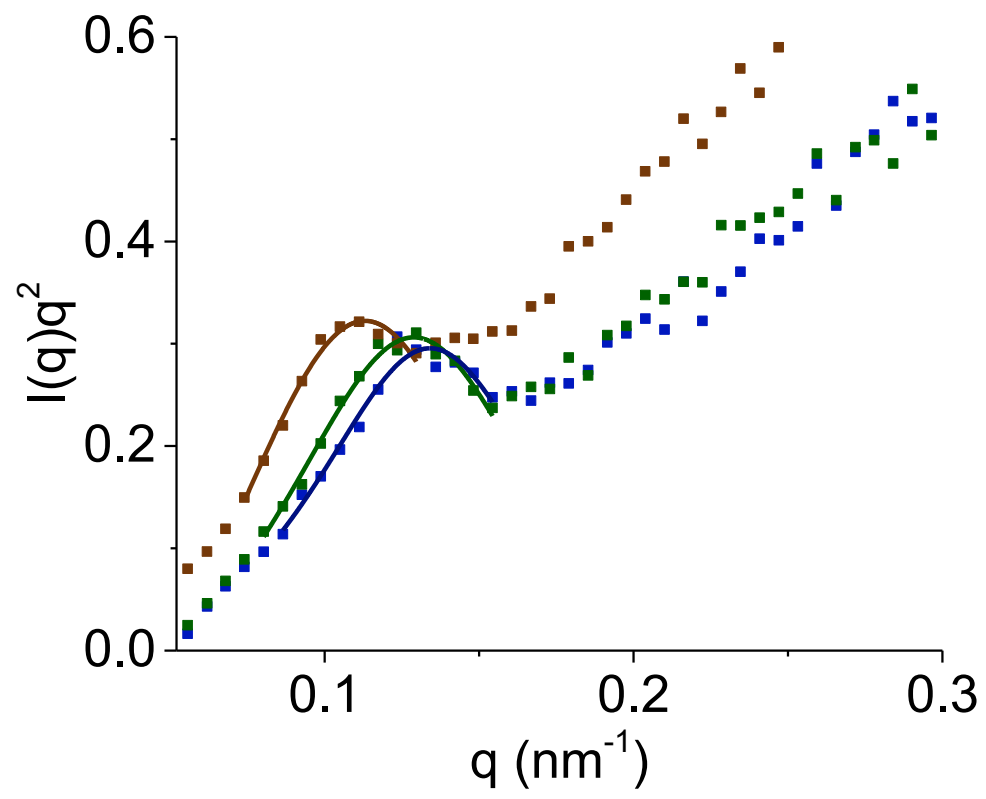

Figure S4.10. Kratky plots of the AuNR-CNC systems (matching for CNCs) containing 0.0014 (brown), 0.007 (green) and 0.021 (blue) wt\% AuNRS and 8 wt\% CNCs, showing Lorentz fits.

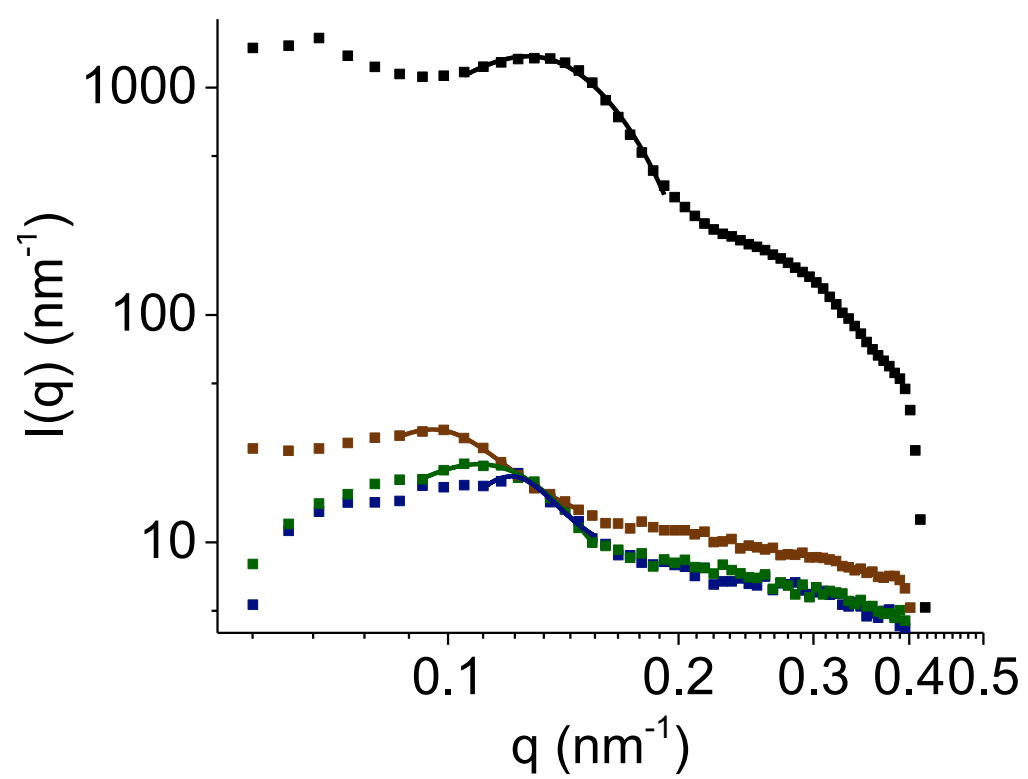

Figure S4.11. SANS plots of the AuNR-CNC systems (matching for CNCs) containing 0.0014 (brown), 0.007 (green) and 0.021 (blue) wt\% AuNRS and 8 wt\% CNCs, showing Lorentz fits. An 8 (black) wt\% CNC curve is shown for comparison. 


\section{Correlation distances}

Table S1.1. Correlation distances obtained from a Lorentz fit of the structure peak of AuNR-CNC systems containing 1-10.6 $w t \%$ of CNCs and 0.0014 wt\% of AuNRs. The correlation distance reduces as the CNC concentration increases.

\begin{tabular}{cccccccc}
\hline $\begin{array}{c}\text { CNC conc. } \\
\text { (wt\%) }\end{array}$ & $\begin{array}{c}\text { AuNR } \\
\text { conc. } \\
\text { (wt\%) }\end{array}$ & $\begin{array}{c}\mathbf{q}_{\max }\left(\mathbf{n m}^{-1}\right) \\
\text { Ch. nem. } \\
\text { phase }\end{array}$ & $\begin{array}{c}\text { St. dev. } \\
\left(\mathbf{n m}^{-1}\right)\end{array}$ & $\begin{array}{c}\mathrm{d}(\mathbf{n m}) \\
\text { Ch. nem. } \\
\text { phase }\end{array}$ & $\begin{array}{c}\mathbf{q}_{\max }\left(\mathrm{nm}^{-1}\right) \\
\text { lso. phase }\end{array}$ & $\begin{array}{c}\text { St. dev. } \\
\text { (nm }\end{array}$ & $\begin{array}{c}\mathbf{d} \text { (nm) } \\
\text { Iso. } \\
\text { phase }\end{array}$ \\
\hline 8 & - & 0.14593 & 0.00026 & 43 & - & & - \\
\hline 5 & 0.0014 & 0.10268 & 0.00056 & 61 & 0.10644 & 0.00066 & 59 \\
8 & 0.0014 & 0.11852 & 0.00046 & 53 & 0.11757 & 0.00052 & 53 \\
10.6 & 0.0014 & 0.12174 & 0.00060 & 52 & 0.12168 & 0.00043 & 52
\end{tabular}

Table S1.2. Correlation distances obtained from a Lorentz fit of the structure peak of AuNR-CNC systems containing 0.0014$0.021 \mathrm{wt} \%$ of AuNRs and $8 \mathrm{wt} \%$ of CNCs.

\begin{tabular}{ccccc}
\hline CNC conc. (wt\%) & $\begin{array}{c}\text { AuNR conc. } \\
(\mathbf{w t} \%)\end{array}$ & $\mathbf{q}_{\max }\left(\mathbf{n m}^{-1}\right)$ & St. dev. $\left(\mathbf{n m}^{-\mathbf{1}}\right)$ & $\mathbf{d}(\mathbf{n m})$ \\
\hline 8 & - & 0.14593 & 0.00026 & 43 \\
\hline 8 & 0.0014 & 0.11826 & 0.00060 & 53 \\
8 & 0.007 & 0.13015 & 0.00036 & 48 \\
8 & 0.021 & 0.13562 & 0.00026 & 46
\end{tabular}

Table S1.3. Correlation distances obtained from a Lorentz fit of the structure peak of AuNR-CNC containing 1-10.6 wt\% of CNCs and 0.0014 wt\% of AuNRs.

\begin{tabular}{ccccc}
\hline CNC conc. (wt\%) & $\begin{array}{c}\text { AuNR conc. } \\
\text { (wt\%) }\end{array}$ & $\mathbf{q}_{\max }\left(\mathbf{n m}^{-\mathbf{1}}\right)$ & St. dev. $\left(\mathbf{n m}^{-\mathbf{1}}\right)$ & $\mathbf{d}(\mathbf{n m})$ \\
\hline 8 & - & 0.14593 & 0.00026 & 43 \\
\hline 8 & 0.0014 & 0.11288 & 0.00110 & 56 \\
10.6 & 0.0014 & 0.13843 & 0.00147 & 45
\end{tabular}

Table S1.4 Correlation distances obtained from a Lorentz fit of the structure peak of Au-CNC containing 0.0014-0.021 wt\% of AuNRs and 8 wt\% of CNCs.

\begin{tabular}{ccccc}
\hline CNC conc. (wt\%) & $\begin{array}{c}\text { AuNR conc. } \\
\text { (wt\%) }\end{array}$ & $\mathbf{q}_{\max }\left(\mathbf{n m}^{-1}\right)$ & St. dev. $\left(\mathbf{n m}^{-1}\right)$ & $\mathbf{d}(\mathbf{n m})$ \\
\hline 8 & - & 0.14593 & 0.00026 & 43 \\
\hline 8 & 0.0014 & 0.11302 & 0.00099 & 56 \\
8 & 0.007 & 0.12882 & 0.00070 & 49 \\
8 & 0.021 & 0.13409 & 0.00139 & 47
\end{tabular}


SANS data of the isotropic and nematic phases. Comparison of SANS curves obtained from the isotropic and nematic phases of the AuNRs and CNCs mixtures.

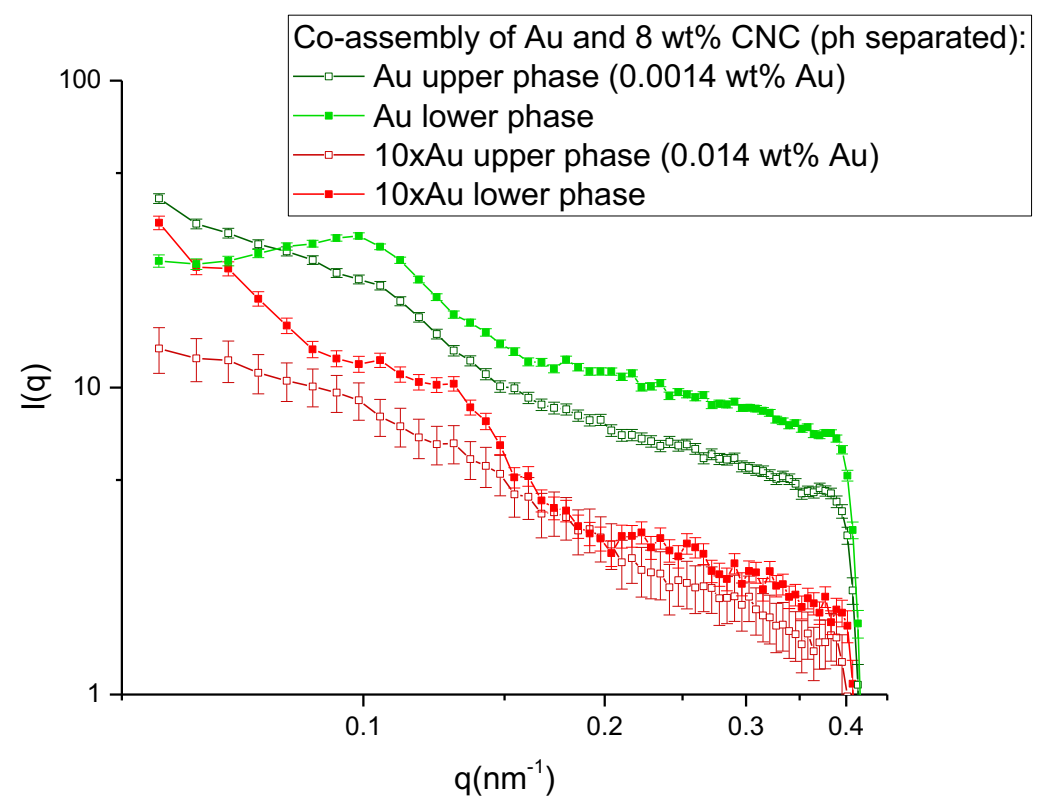

Figure S5. SANS curves of Au-CNC assemblies containing 8 wt\% CNCs and 0.014 wt\% AuNRs (red curves), 0.0014 wt\% AuNRs (green curves). A comparison of the upper (isotropic) and lower (cholesteric) phases are shown. The influence of the concentration of gold nanorods on the position of the structure peak is also clearly visible.

Summary of correlation distances of CNCS and AuNRs in the assemblies as function of concentrations.
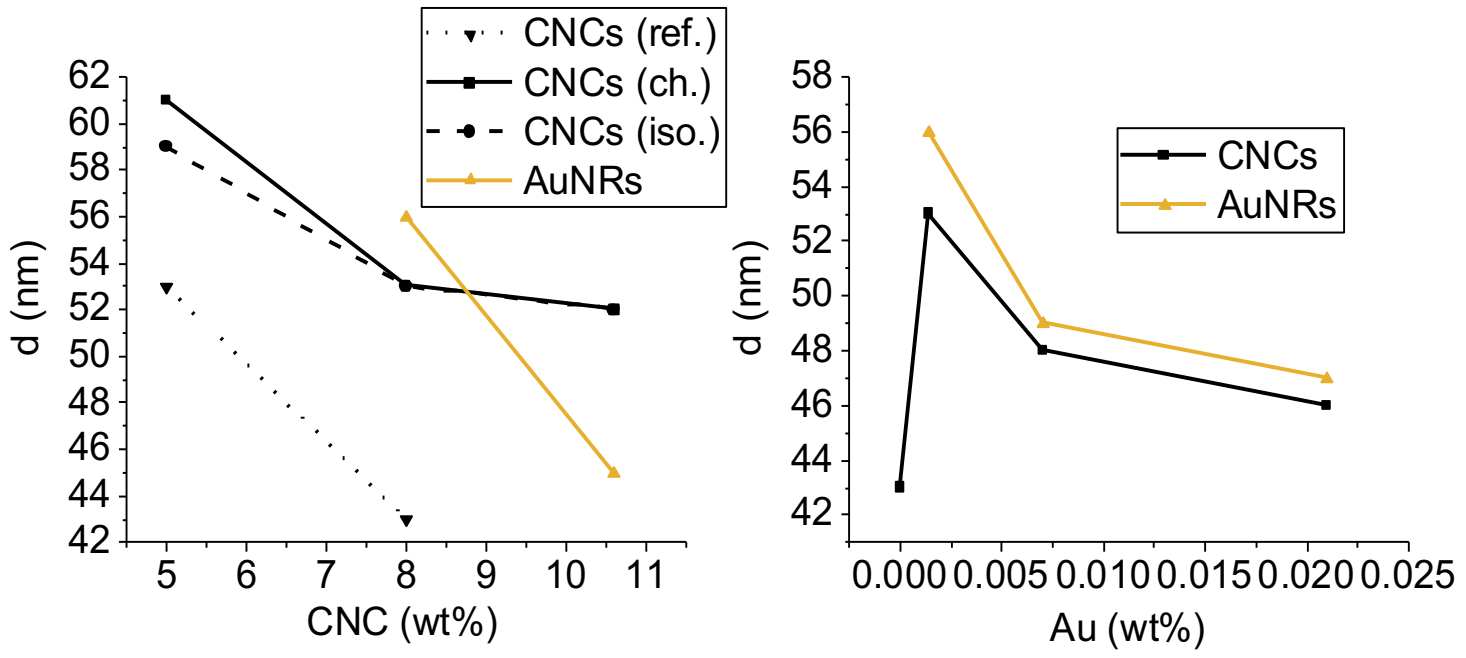

Figure S6. Evolution of the correlation distances between AuNRs and CNCs in the investigate AuNR-CNC systems as a function of the CNC (left) and AuNR (right) concentrations. 


\section{References}

1 L. Jasmani, S. Eyley, R. Wallbridge and W. Thielemans, Nanoscale, 2013, 5, 10207-11.

2 L. Scarabelli, A. Sánchez-Iglesias, J. Pérez-Juste and L. M. Liz-Marzán, J. Phys. Chem. Lett., $2015,6,4270-4279$.

3 M. Martínez-Sanz, M. J. Gidley and E. P. Gilbert, Soft Matter, 2016, 12, 1534-1549.

4 G. Nyström, M. P. Fernández-Ronco, S. Bolisetty, M. Mazzotti and R. Mezzenga, Adv. Mater., 2016, 28, 472-478.

5 P. Strunz, K. Mortensen and S. Janssen, Phys. B Condens. Matter, 2004, 350, E783-E786.

$6 \quad$ M. Uhlig, A. Fall, S. Wellert, M. Lehmann, S. Prévost, L. Wågberg, R. von Klitzing and G. Nyström, Langmuir, 2016, 32, 442-450.

7 C. Schütz, M. Agthe, A. B. Fall, K. Gordeyeva, V. Guccini, M. Salajková, T. S. Plivelic, J. P. F. Lagerwall, G. Salazar-Alvarez and L. Bergström, Langmuir, 2015, 31, 6507-6513.

8 U. Keiderling, Appl. Phys. A Mater. Sci. Process., 2002, 74, s1455-s1457.

9 C. A. Schneider, W. S. Rasband and K. W. Eliceiri, Nat. Methods, 2012, 9, 671-675.

10 D. Nečas and P. Klapetek, Cent. Eur. J. Phys., 2012, 10, 181-188. 